\title{
LES SÉQUENCES TÉLÉVISUELLES
}

\section{Noël Nel ${ }^{1}$}

La théorie de la télévision qui commence à s'éloigner de la fascination du "mauvais objet" comme des généralisations abusives portées par les scénarios catastrophistes (manipulation, décervelage, sidération) se met à considérer frontalement la question de la complexité du média. Ce faisant, un certain nombre de concepts indispensables à l'avancée de la réflexion se trouvent explorés et travaillés dans des perspectives qui tiennent peu à peu compte, et de la spécificité du média, et des évolutions décisives des méthodologies dans le sens de la liaison désormais indispensable entre texte (émission, produit, œuvre) et contexte. Cependant, il nous manque à l'évidence une proposition (au moins) qui permettrait d'entrevoir la manière dont est structurée et composée une émission de télévision. Entre le niveau micro-textuel du plan et le niveau macro-textuel de l'ensemble du texte télévisuel, que se passe-t-il ? Comment segmenter ou fragmenter une émission? Quels instruments utiliser pour fonder une typologie de base des séquences télévisuelles capable de rendre compte de la diversité des situations en même temps que de l'hétérogénéité constitutive du média?

\footnotetext{
1 Professeur à l'Université de Metz. Fondateur du CREM (Centre de recherche sur les médias) et co-directeur de celui-ci.
} 
Répondre à ces questions, c'est donc combler une lacune et c'est aussi, comme on le verra, engager une forte relativisation de la place du narratif dans l'activité de symbolisation de la télévision.

\section{L'agir télévisuel comme acte de symbolisation}

L'agir télévisuel est l'activité déployée par le média télévision dans le cadre de ses régimes d'action, matérielle (technique, économique) et symbolique. Le dernier aspect peut s'appréhender aux différents niveaux de l'interaction médiatique en société, de la programmation globale d'une chaîne, de l'action de chaque émission particulière, des séquences qui la composent et enfin des actes particuliers qui s'y développent. Il sera ici envisagé dans une perspective transversale, au niveau global.

\section{Un modèle de l'agir télévisuel}

Il m'est arrivé de proposer une définition en extension de l'agir télévisuel dans le champ médiatique ${ }^{1}$, en regroupant les pratiques communicationnelles complexes et les logiques différentes à l'œuvre sous un certain nombre de catégories fondamentales. Partant du schéma d'A. Gosselin qui concernait la communication politique ${ }^{2}$, il m'est apparu nécessaire de le transférer à un autre type de communication. De cette façon, en reformulant et A. Gosselin, et les catégories de Max Weber, Goffman et Habermas, j'ai cru pertinent de repérer quatre grands types d'agir communicationnel à la télévision : l'agir téléologique par habitus, l'agir axiologique, l'agir affectuel, l'agir dramaturgique et spectaculaire. L'agir télévisuel ne se limite pas à la sacro-sainte trilogie fonctionnelle des origines : informer, éduquer, distraire. Il n'est pas sûr que chaque type d'agir nommé ait produit des genres d'émissions parfaitement constitués. Mais on fait l'hypothèse qu'il existe, au-delà du niveau micro-textuel du simple acte de discours à l'œuvre dans un moment d'émission, un niveau intermédiaire baptisé séquence (on pourrait dire aussi segment ou

1 N. NEL, "Généricité, séquentialité, esthétique télévisuelles", Réseaux n 81 , janvierfévrier 1997, p. 33-46.

2 A. Gosselin, "La communication politique. Cartographie d'un champ de recherche et d'activités", Hermès n 17-18, 1995. 
fragment) dont l'unité et la cohérence reposent sur un type dominant d'agir. On aboutit ainsi à une stratification de l'agir à trois niveaux qui peuvent cohabiter ou s'enchâsser : actes de discours, séquence intermédiaire d'agir, agir global . Et on postule que l'agir téléologique par habitus repose sur la prédominance de séquences informatives, culturelles, distractives; que l'agir axiologique affectionne les séquences implicatives; que l'agir affectuel mobilise les séquences expressives; que l'agir dramaturgique passe par des séquences performatives spectaculaires. En ce sens, l'agir global d'une émission, à titre de visée stratégique relevant d'une ligne éditoriale explicite ou comme résultante de combinaisons effectivement à l'œuvre, est protéiforme.

\section{La nature séquentielle du texte télévisuel}

Après avoir défini l'agir télévisuel dans une perspective de complexité, il convient sans doute d'expliciter la conception que l'on propose d'une émission de télévision qui serait pensée, sur le plan compositionnel, dans le cadre d'un modèle de l'hétérogénéité. Un tel modèle, proche de celui de J.-M. Adam pour les discours écrits et oraux $^{1}$, doit offrir plusieurs garanties : ajouter au plan de la seule structuration un plan de la configuration pragmatique, de façon à relier texte et contexte; opérer une distinction entre niveau textuel global et niveau séquentiel intermédiaire; rendre compte des modes de combinaison / transformations entre séquences. En somme, il doit fonctionner en modèle des plans d'organisation d'une émission télévisuelle en permettant d'appréhender la nature compositionnelle complexe et l'hétérogénéité relative de toute émission.

On reprend donc l'idée que toute émission de télévision est faite de propositions liées dotées d'une configuration pragmatique. Les propositions sont des séquences bénéficiant d'un liage ou d'une syntaxe. La configuration pragmatique est la construction d'une vision de monde (factuel, fictionnel, virtuel) à cohésion plus ou moins forte, vision dotée d'un ancrage énonciatif repérable et portée par un agir global visant la cohérence. Les séquences apparaissent donc comme la base de l'édifice, les unités minimales de la composition,

1 J.-M. ADAM, Les textes: types et prototypes, Paris, Nathan Université, 1992. 
les formes élémentaires prototypiques, disponibles pour de multiples combinaisons et dont l'ampleur est variable.

\section{Actes illocutoires et séquences télévisuelles}

Comme les typologies des actes de discours sont loin d'être unifiées, force est de préciser de quelle construction l'on se réclame, en n'oubliant jamais qu'un acte discursif passant par le langage et une action portée par une médiation technico-symbolique ne se recouvrent pas parfaitement. Prenons la théorie de J.R. Searle, qui distingue cinq grands types d'actes illocutoires :

Nous disons à autrui comment sont les choses (assertifs), nous essayons de faire faire des choses à autrui (directifs), nous nous engageons à faire des choses (promissifs), nous exprimons nos sentiments et nos attitudes(expressifs) et nous provoquons des changements dans le monde par nos énonciations (déclaratifs) ${ }^{1}$.

Il est possible de répartir les actes illocutoires télévisuels dans les cinq catégories de la taxinomie de Searle, pour peu que l'on précise auparavant un point concernant l'énonciation télévisuelle. En tant que faire communicationnel adressé à un téléspectateur qui co-construit, elle est action complexe qui engage en fait trois actions cooccurrentes. Elle est production et diffusion d'un discours télévisuel, dans le cadre spécifique de la présentation et d'un réseau de réception en instantanéité et simultanéité. Elle est implication de l'énonciataire. Elle est provocation de celui-ci à réagir. Si bien qu'il est nécessaire de croiser en quelque sorte les cinq actes illocutoires et les trois niveaux d'actions. Sans dérouler dans sa totalité le tableau récapitulatif qui en résulte, on se contentera d'en souligner les points majeurs.

Les actes assertifs (citer, expliquer, insister, rappeler) passent prioritairement par les séquences explicatives et les séquences répétitives. Les actes directifs poussent à faire raisonner (comprendre, supposer, deviner, juger) et à émouvoir selon les multiples registres disponibles (amuser, troubler, faire admirer, attrister, etc.) : il y faut des séquences argumentatives et des séquences performatives. Assez rares à la télévision, les actes promissifs institutionnels interviennent

1 J. R. SEARLE, Sens et expression. Études de théorie des actes de langage, Éd. de Minuit, 1982, p. 32. 
dans les séquences du même nom (annonces de programme, par exemple). Qu'ils soient manifestés par les journalistes qui émettent des opinions ou par des invités qui expriment leur émotion, les actes expressifs composent donc les séquences expressives. Les actes déclaratifs semblent les plus nombreux :

La caractéristique définitionnelle de cette classe consiste dans le fait que l'accomplissement réussi de l'un de ses membres provoque la mise en correspondance du contenu propositionnel avec la réalité ; l'accomplissement réussi garantit que le contenu propositionnel correspond au monde (...) Les déclarations provoquent une modification du statut ou de la situation de l'objet ou des objets auxquels il est fait référence seulement en vertu du fait que la déclaration a été accomplie avec succès ${ }^{1}$.

Les actes déclaratifs interviennent notamment dans la construction de l'émission (préparer, structurer, ajouter, compléter) et dans l'implication de l'énonciataire (s'adresser à lui, focaliser). La préparation du texte télévisuel, en raison de l'inévitable sérialité, entraîne l'existence d'opérations transtextuelles. La structuration du texte engage notamment la question des relations entre séquences élémentaires (liaison, alternance, enchâssement), question à aborder en un second temps. Ajouts et compléments passent par des séquences complétives. Quant à l'implication de l'énonciataire, elle mobilise à la télévision la séquence conversationnelle, plus exactement le monologue de l'adresse au téléspectateur.

\section{Proposition d'une typologie de base des séquences TV}

De l'essai d'inventaire qui vient d'être dressé, à partir de démarches explicites, on peut dégager un nombre restreint de séquences élémentaires. Au registre des séquences de base constituant le premier niveau, on retient :

- les séquences conversationnelles: sous la forme monologale de l'acte déclaratif d'adresse (acte de présentation), mais encore sous la forme dialogale et polylogale ;

- les séquences descriptives avec leurs actes assertifs ;

1 Ibid., p. 56-57. 
- les séquences actionnelles ${ }^{1}$ qui se proposent de dépeindre, rapporter, relater, programmer, raconter ;

- les séquences explicatives avec leurs actes assertifs, séquences principales ou séquences complétives ;

- les séquences performatives : séquences argumentatives à actes directifs, séquences implicatives à actes directifs et déclaratifs.

A un second niveau, on peut au moins mentionner :

- les séquences interstitielles du programme global, séquences promissives (bandes-annonces), séquences directives et esthétiques (publicité institutionnelle et commerciale);

- les séquences transtextuelles et réflexives : séquences didactiques sérieuses de retour sur les archives, séquences ludiques de détournement de dispositifs d'information ;

- les séquences esthétiques de l'art vidéo.

Dans les lignes qui suivent, il s'agit d'analyser en détail le fonctionnement de ce qui a été appelé le niveau de base de la séquentialité télévisuelle.

\section{Les séquences télévisuelles élémentaires}

Toutes les séquences élémentaires qui ont été repérées sont des ensembles de dimensions variables, en position d'autonomie (totale ou relative) ou de dépendance, présents dans toutes les émissions télévisées, le plus souvent à ce niveau intermédiaire entre le simple acte de discours et l'ensemble de l'émission.

\section{La séquence conversationnelle}

Il faut commencer par elle parce que la télévision situe une partie de sa spécificité dans le régime scopique de la présentation ${ }^{2}$, où la parole est reine. La présentation télévisuelle ${ }^{3}$ est fondamentalement

1 Je rejoins la mise au point de F. REvaz, Les textes d'action, Metz, Université de Metz, 1997. Elle propose une judicieuse remise en cause de la priorité accordée au narratif et une redistribution de celui-ci en discours actionnels incluant, à une place plus restreinte, les discours narratifs.

2 N. NEL, "Les régimes scopiques" 1 et 2, Champs visuels, $\mathrm{n}^{\circ} 1$ et 2 , janvier et mars 1996.

3 La description la plus pertinente à mes yeux est celle de J.-P. EsquenazI, Le pouvoir d'un média. TF1 et son discours, Paris, L'Harmattan, 1996. 
une énonciation centrale ancrée à un site (le studio), définissant une fonction(présentateur, animateur) qui est codifiée par des règles discursives, dont la règle du direct, et qui produit un espace public, actuel (l'écran) et virtuel (la société) à la fois. Énonciation centrale : la parole du présentateur, qui est incarnée dans un corps visible, qui passe par un regard "les yeux dans les yeux", marque d'identification et caution de référenciation, cette parole définit le régime de la présentation comme acte omniprésent émanant d'un corps ancré à un site. Par défaut, il s'agira seulement d'une voix acousmatique annonçant des programmes ou commentant une émission (le $81 / 2$ ou le bulletin météo d'Arte). Le présentateur est donc un annonceur institutionnel, régisseur général de l'information, animateur d'émissions sérieuses ou ludiques. Sa fonction est attachée à un site (le studio et le plateau) devenu un lieu, au double sens d'indicateur de stabilité (M. de Certeau) $)^{1}$ et de milieu (P. Boudon) ${ }^{2}$. Vrai ou faux, et davantage fait sémiotique et social que fait purement technique, "le direct transforme le présent en présence, il le spectacularise et le voue à une forme d'existence en dehors du temps"3. Enfin, ce site de la présentation est l'interface absolu entre espace public actuel du studio et espace virtuel du social, entre l'émission actuellement diffusée et toutes les autres émissions à générer. Toutes les commutations entre studio et monde, tous les glissements d'une émission à l'autre se font en ce point central, autour de l'axe fixe du regard $Y-Y$, ou en un espace abstrait rattachable à ce point de référence.

Dans ce site de la présentation ou en relation avec lui, se déploient les grands types de la parole médiatique, que l'on peut, pour des raisons d'intelligibilité, réduire à trois ${ }^{4}$ : parole de découverte qui expose à travers l'énonciation experte le savoir savant; parole de construction qui exprime le savoir d'opinion à travers la proclamation individuelle ou l'interrelation contradictoire ; parole d'émergence qui fraye un chemin à l'inconscient du sujet anonyme ou célèbre.

1 M. DE CERTEAU, L'Invention du quotidien, Paris, Gallimard, 1990, p. 173.

2 P. Boudon, Introduction à une sémiotique des lieux, Presses de l'Université de Montréal, Klincksieck, 1981. Il y propose une théorie du lieu comme espace pluridimensionnel : espace mythologique, espace de rituels, espace social, espace architectural.

3 J.-P. ESQUENAZI, op. cit., p. 123.

4 P. Charaudeau et R. Ghiglione, La parole confisquée. Le talk show, Paris, Dunod, 1997. P. Charaudeau, Le discours d'information médiatique, Paris, Nathan/INA, 1997, p. 208. 
La séquence conversationnelle est d'abord monologale. Parole de présentation, elle signifie toujours discours adressé. C'est la parole du présentateur animateur ; c'est la relation ou reconstitution historique d'un Alain Decaux ; c'est le point de vue personnel d'un Michel Polac (Trois minutes pour faire lire) ou d'un Olivier Barrot (Un livre, un jour). Selon les visées et les thèmes qu'elle envisage, cette parole monogérée peut aisément infléchir la séquence monologale descriptive ou actionnelle vers une autre séquence, explicative par exemple. Par contre, dès qu'on entre dans la séquence dialogale et polylogale, s'impose alors la structuration inhérente à la conversation polygérée. Certes, comme l'ont relevé Jakobson, Benveniste et d'autres ${ }^{1}$, la coénonciation est déjà dans le texte conversationnel monogéré. Si la terminologie est loin d'être fixée en ce qui concerne les genres ou les formats (interview, entretien, duel ou face à face, débat, discussion), par contre l'accord semble trouvé sur l'insertion nécessaire de la séquence dans un modèle hiérarchique et dynamique du texte conversationnel. Ce dernier est pensable comme ensemble d'échanges (corps de l'interaction) encadrés par des séquences monologales d'ouverture et de clôture plus ou moins ritualisées par les contraintes sociales. Chaque séquence d'échange est une suite d'interventions -actes de langage ou clauses- qui sont les unités minimales de comportement entrant dans une structure binaire (intervention initiative ou réactive) ou une structure ternaire (initiative / réactive / évaluative). La séquence conversationnelle dialogale trouve alors son unité dans la stabilité ou le changement d'orateur et de thème. Et lesdites séquences sont liées ou enchâssées dans l'ensemble conversationnel.

\section{La séquence descriptive}

La question de la description audiovisuelle est d'une effroyable complexité et attend encore une clarification acceptable ${ }^{2}$. Elle pose les

1 R. JAKoBson, Eléments de linguistique générale, 1963, p. 30: "Tout discours individuel suppose un échange". E. BENVENISTE, Problèmes de linguistique générale, 1974, p. 85 : "Le monologue est un dialogue intériorisé, formulé en langage intérieur, entre un moi locuteur et un moi écouteur".

2 Je m'appuie essentiellement, en les suivant ou en les reformulant, sur: J. Fontanille, "La description au cinéma", Degrés, n 64, 1990 ; A. GARdiES, "Tu n'as rien vu à Manhattan", Génériques, n 1, Hiver 1994, Lyon., p. 47-57; A. GARDiEs, " $L$ 'œil était dans le monde. L'émergence du point de vue", Génériques, n² 2, printemps-été 1995, Lyon, p. 5-13 ; C. MANCINI, "Retour sur le descriptif. Revoir Hiroshima", Génériques, n 4, Lyon, p. 33-39. 
difficiles problèmes attachés aux grandes catégories constitutives. Problèmes des relations entre sémiotique du monde naturel et sémiotique construite : la description serait-elle la simple retransmission mimétique du mouvement inhérent aux actions sensori-motrices et événements (une perception descriptive), et la narration en serait-elle l'intellection? La sémiotique audiovisuelle incruste une sémiotique naturelle dans une sémiotique construite : par quels phénomènes cela se traduit-il ? Faut-il postuler une compétence d'observation avec un rôle d'observateur? Problèmes, aussi, de la distinction entre narratif et non narratif, ce dernier étant trop vite assimilé au descriptif, au sein d'une opposition binaire, alors qu'il est souhaitable de se tourner vers des distinctions scalaires. Problème, enfin, des relations entre énoncé et énonciation : c'est la question de la tension discursive et, au-delà, de la théorie de l'énonciation audiovisuelle (cinématographique et télévisuelle). La tension entre narratif et non narratif, énoncé et énonciation, est d'autant plus marquée qu'entre la séquence descriptive et le reste s'installent des démarcations franches assimilables souvent à des pauses de la narration, et que la séquence descriptive est détendue, comme à contre-courant, alors que le mouvement perdure toujours un peu dans l'image animée. Si bien que des degrés descriptifs doivent être envisagés, de la description retenue (pano ou recadrage descriptifs) à la description diffuse (planséquence), dispersée (plan de coupe descriptif) et compacte (séquence descriptive). Ces trois problèmes sont loin d'avoir trouvé une solution correcte.

Cependant, la description audiovisuelle offre la particularité de faire appréhender par les sens l'existence plus ou moins marquée d'une présence observatrice. Fontanille la décrit comme effet de sens qui introduit un observateur, une sorte de foyer conceptuel (maître du point de vue, centre de perspective ou d'orientation) qui observe l'espace, l'action ou l'événement, et dont les opérations d'observation sont inscrites dans le texte. Cet observateur peut, soit rester un filtre minimal, implicite et abstrait, soit être pris en charge par l'énoncé (voix de narrateur, caméra subjective d'assistant participant, au titre de simple figurant ou de protagoniste). Une première difficulté tient au fait qu'il faut en passer par la relation sujet / objet, laquelle permet, dans certaines circonstances, à partir de la modalisation de l'objet regardé, d'inférer la présence d'un sujet observateur. Une deuxième difficulté est que le sujet peut se subdiviser en narrateur (la voix), observateur (le regard), focalisateur (la conscience), les trois rôles se 
combinant dans des proportions variables. Une dernière difficulté est que le point de vue peut allier plusieurs dimensions, perceptive (description-constat, monstration), idéologique (description-révélation de cadres de pensée), narrative (description prolongeant l'action), esthétique (description - contemplation). L'observation est moins discutable lorsque s'impose l'acte de vouloir, pouvoir, savoir décrire, dans une continuité syntagmatique, acte à distinguer de la simple monstration en ce qu'il est regard construit ostensif, parcours rythmé qui peut être désancré ou déconnecté d'une instance diégétique. Mais il reste à distinguer ce que Mancini nomme le régime descriptif de la monstration, cette description statique et lente à faible densité informative qui pourrait être l'œuvre d'un observateur "oisif" (Fontanille), et ce qu'elle nomme le régime narratif de la monstration, cette description à effet de narrativité, à rythme plus soutenu et à densité informative plus forte.

Il faut cependant aller plus loin encore dans l'analyse pour parvenir à décrire correctement la macro-structure de la séquence descriptive. Sur le plan littéraire, la description est discours hiérarchisé qui mobilise quatre procédures rappelées par J.-M. Adam. L'ancrage : un thème-titre est institué d'emblée, ou affecté au final. Le travail d'aspectualisation s'engage par prédication de propriétésqualités (forme, taille, etc.) et décorticage en parties (synecdoque). La mise en relation s'établit par prédication de traits de situation (métonymie spatiale, métalepse temporelle) et par relations associatives (comparaison, métaphore). Les sous-thématisations dérivées peuvent s'ouvrir, ainsi que les reformulations qui opèrent des réancrages. Tel est le mécanisme langagier de la description. A. Gardies défend l'idée que la séquence descriptive cinématographique ne ressemblerait pas à l'organigramme stratifié de la séquence langagière en ce qu'elle ne comporterait qu'addition et juxtaposition. Cela reste à démontrer. Dans le cas de la télévision, que serait véritablement la séquence descriptive ? Elle n'est pas le simple geste de monstration et de présentation par l'image du mouvement du monde. Elle n'est pas l'acte rapide en plan unique, insert descriptif ou pano descriptif, dont $M$. Colin disait qu'il opère nécessairement en sens inverse du mouvement habituel, donc de droite à gauche ${ }^{1}$. Les retransmissions en direct non préparé, dans le cas d'événements soudains et exceptionnels,

1 M. Colin, "La dislocation", in Aumont et LEUTRAT, Théorie du film, Albatros, 1980, p. 71-91. 
conduisent-elles à des séquences descriptives? L'étude de la "couverture médiatique" de la "révolution roumaine" (décembre 89 janvier 90) par les chaînes françaises a conduit F. Jost à déclarer que, face aux images directes, "le présentateur était incapable de dépasser le stade de la description indéfinie modalisée"1 et que "la description indéfinie se dévide sans logique causale, sans échapper, même à la contradiction. Le commentaire est aphasique, il se contente d'épeler les phénomènes" (Kant). Jost ajoute encore: "Ce que montre la verbalisation des images par le commentaire, c'est que, sans la connaissance préalable de la réalité filmée, la succession et la transformation mises en jeu par l'image animée n'accèdent qu'à la description et non au récit".

Si certaines remarques de ces analyses sont pertinentes, par contre certains points prêtent à discussion. Pour tenter de les dépasser, prenons l'exemple de Brut (B. Doutriaux et P. Ouazan), magazine multithématique composé de séquences entrecoupées par un fond noir. Quelques-unes sont commentées par leur opérateur (photographe, JRI) qui expose l'angle de compréhension qu'il souhaiterait, ou par une voix off qui révèle les informations contextuelles sans lesquelles tout resterait obscur. Le plus généralement, les séquences sont fidèles au titre général : des images sans commentaires, une moitié produite par la chaîne Arte, une autre moitié issue de rushes achetés. Peu de montage: un discret enchaînement de plans-séquences, quelques "avances rapides" pour signifier qu'il y a eu ellipse. Les séquences "brutes" sont-elles descriptives ? Prenons la séquence sur le grand cuisinier Guy Savoy (11-07-97). Nous le découvrons en action, au cœur de sa cuisine, organisant les priorités de cuissons, supervisant l'ordre des livraisons en salle, évaluant les plats confectionnés par les marmitons, prenant au pied levé les décisions de suppléance. La caméra varie un peu les points de vue tout en restant à proximité du chef, et le son direct parfois inaudible est un garant d'authenticité. Incontestablement, les séquences "brutes" montrent avec ostension des actions du monde, en respectant d'ailleurs les trois règles classiques de l'unité de lieu, de temps, d'action. Elles relèvent de choix subjectifs qui renvoient au monde du travail, aux échanges humains, aux fonctionnements institutionnels, en privilégiant le côté atypique des choses : ce qui est insolite, parfois extraordinaire. Bref, la séquence de Brut est plutôt

1 F. Jost, Un monde à notre image , Paris, Méridiens-Klincksieck, 1992, p. 35-36. 
une séquence actionnelle de relation qu'une séquence descriptive. Elle postule que l'image est dotée d'un régime d'intelligibilité qui peut parfois se passer du commentaire. On retrouve les mêmes caractéristiques dans le reportage de Zoom (A. Harris et A. de Sédouy) consacré, à l'époque, aux événements de Mai 68. Après une séquence conversationnelle de plateau où les deux présentateurs annoncent qu'ils ouvrent un débat exceptionnel pour un événement exceptionnel, un reportage nous est proposé, déclaré à la fois incomplet et "sans concession". Nous assistons à une sélection de scènes, moments et faits, qui ne privilégie pas un héros unique. Une voix off, "blanche" et épisodique, donne les repères temporels et délivre les informations utiles à la compréhension des scènes. La caméra est constamment au cœur de l'événement, y compris lors des charges policières. Séquence de relation, à accents de chronique, mais pas description!

Que faut-il alors pour que l'on puisse parler sans hésiter de séquence descriptive ? L'exemple célèbre du mariage du roi Baudouin et de la reine Fabiola commenté par Léon Zitrone nous fournit une sorte de clé. Dans ce cas, nous avons la certitude que le commentaire de l'événement a été minutieusement préparé. Les images du reportage, du moins en son début, montrent le drapeau belge, puis l'extérieur immédiat du palais royal de Bruxelles et l'intérieur de la salle du trône: partout, l'attente patiente! Le commentaire qui accompagne continûment les images en plans-séquences délivre un flot d'informations tirées de fiches préparatoires. Il est aisé d'y repérer la structure descriptive. Ancrage du thème-titre : le mariage royal "à $289 \mathrm{~km}$ au nord-est de Paris" (sic). Aspectualisations constantes concernant le palais royal et les deux héros du jour. Décorticage en synecdoque : on passe du palais à l'histoire de celui-ci ou à la salle du trône qu'il contient et à ses lustres; on passe des royaux protagonistes à leur famille et à l'histoire de la Belgique. En cette situation initiale d'attente, l'asynchronisme gouverne le lacis audiovisuel ; le point de synchronisme surgit lorsqu'entrent les invités et le cortège royal. Bref, la séquence descriptive requiert d'abord l'intentionnalité de l'acte global de description, à distinguer de l'acte épisodique local. Elle exige la forme structurante du dispositif symbolique hiérarchisé, proche parfois du catalogue ou de la litanie. Il lui faut la meilleure synergie entre dimension verbale du commentaire et dimension audiovisuelle de la mise en représentation. De ce point de vue, on peut maladroitement commenter des images d'un direct non préparé (charnier de Timisoara, débarquement sur la Lune ou sur 
Mars), vouloir coller par la parole à ce qui est montré sans structurer en rien une séquence descriptive. Celle-ci réclame intention et forme adéquate, sans doute aussi culture et distance chez le commentateur. Il semble donc indispensable de distinguer fonction descriptive de la parole, de l'image et du son, et séquence descriptive.

\section{Les séquences actionnelles}

Il peut paraître également nécessaire d'opérer une distinction entre (re)présentation d'événements et (re)présentation d'actions. Même si les deux notions sont assez fréquemment confondues, elles doivent être reconsidérées à la lumière des débats des années 55-60, et des propositions de Wittgenstein, Austin, Ricœur. Il suffira d'indiquer que le réseau conceptuel de l'événement est celui de la cause, la loi, le fait, quand celui de l'action est celui des intentions, des motifs ou raisons d'agir, des agents ${ }^{1}$. En reprenant les critères fondamentaux de constitution temporelle (dynamicité, transitionnalité), et les critères de constitution participative (causativité, agentivité) mis en avant par certains théoriciens de la sémantique cognitive ${ }^{2}$, on peut distinguer les prédications d'états (séquences descriptives), les prédications d'événements et les prédications d'actions. La distinction entre événements et actions permet ainsi de déboucher sur une sorte d'échelle scalaire entre descriptif et narratif, en évitant l'opposition simplificatrice.

Proches de la description, et pourtant différents d'elle sont le tableau et le portrait. Le tableau apparaît quand "l'exposition de l'objet est si vive, si énergique qu'il en résulte dans le style une image" (Fontanier). On peut encore ajouter :

Le tableau suppose (...) des traits en nombre restreint et habilement groupés autour d'un motif principal. Par là il diffère de la description ordinaire. Il diffère aussi de la narration en ce sens qu'il ne comporte pas toujours une action; de plus, s'il en comporte une, cette action n'a ni commencement, ni milieu, ni fin; nous la voyons à un moment donné, mais à un seul moment, sinon ce serait un récit ${ }^{3}$.

1 Je reprends à mon compte les mises au point de Françoise Revaz, op. cit.

2 J.-C. VerstigGel et G. DenHiÈre, "Cognition et langage" in Langages, 1990.

3 VANNIER, La clarté française. L'art de composer, d'écrire et de se corriger, Paris, Nathan, 1912, p. 300. 
En somme, le tableau est une forme, une structure, un mode de composition séquentielle qui, s'il peut comporter des actions non organisées en récit, en reste au temps non chronologique de la simultanéité, et nous les présente avec vivacité. Qu'est-ce, par exemple, qu'un bulletin météo à la télévision, sinon un ensemble de deux séquences quasi descriptives présentées sur le mode monologal : une séquence constative sur le temps et les températures de la journée, et une séquence programmatrice prédictive sur le temps à venir, le tout traité avec vivacité au niveau du commentaire (B. Gillot-Pétré) et parfois agrémenté d'un plan séquence descriptif ou d'un tableau ? Il en va de même avec le portrait qui applique ces caractéristiques à des états humains quand le tableau privilégie les états physiques et moraux. En somme, tableau et portrait proposent une (re)présentation d'états animés en recourant, dans le lacis audiovisuel et dans le commentaire, à une forme d'anthropomorphisation, à la présentification, à la reconstitution dynamique, à la modalisation énonciative, tous procédés qui relèvent de l'intention d'animer. Les magazines de réalisateurs Strip tease (J. Libon et M. Lamensh) ou Aléas (G. Follin) offrent de nombreux exemples de portraits. Dans le premier : pas de plateau, pas de présentateur, pas de "fil rouge"; de longs reportages qui permettent de déboucher sur les portraits "bruts" de personnages insolites. Le second présente un dispositif original : générique cocasse, flashes émanant d'une radio de voiture et égrenant des faitsdivers, défilé de paysages à travers un pare-brise, présence de l'écrivain Claude Duneton en collectionneur de coupures de faits divers, et reportages traités en autant de portraits de gens ordinaires victimes de faits dérisoires.

Si tableau et portrait sont les (re)présentations d'états et d'actions simultanés, la chronique est (re)présentation d'actions successives qui ne sont pas forcément liées par des rapports de causalité. Avec elle, on quitte la composition tabulaire pour entrer clairement dans la succession linéaire, l'énumération de faits inscrits dans la succession chronologique. Les variétés de chroniques sont multiples : annales, carnets de voyages, journaux intimes, biographies, nécrologies. On peut y rencontrer de nombreuses actions, une pluralité d'acteurs ou un seul, une trame événementielle, mais pas l'unité d'action reconstruite par un narrateur qui impose sa mise en intrigue. Ainsi, les Chroniques de l'hôpital d'Armentières de D. Karlin, T. Lainé et J. Stechel : 8 mois de tournage et montage, 200 à 300 personnes rencontrées, 10 épisodes d'une heure, un "feuilleton documentaire" concernant les 
patients, les familles, les soignants de l'hôpital d'Armentières, près de Lille. Filmage chronologique dans tous les lieux repérés comme intéressants, montage sur place de situations et de cas traités sur le mode dialogal de l'interview. Un portrait en pied de l'hôpital comme "l'un des derniers lieux prométhéens" de la condition humaine, avec une intention explicite : "ce qui me touche dans l'hôpital, c'est qu'il y passe la vie. J'ai voulu la filmer au rythme du sang qui coule dans nos veines, avec son mélange de professionnalisme, de solidarité, d'angoisse, de pudeurs formidables". Une ligne directrice générale, cependant: de la naissance à la mort; des actes médicaux aux réactions personnelles; quelques personnages principaux, comme Tony, le garçonnet de cinq ans qui sert de "fil rouge" aux dix épisodes; une leçon de dignité et de courage qui veut susciter réflexions et réactions. L'ambition d'un mémorialiste du quotidien et de l'ordinaire ${ }^{2}$ ! La chronique peut concerner tous les sujets. Elle peut se faire carnet de voyage comme dans la série documentaire de F. Gall et B. d'Abrigeon Des trains pas comme les autres : un périple à travers le monde, des séquences souvent très descriptives au niveau visuel, le refus de faire parler les habitants, le choix d'un point de vue qui exclut certaines dures réalités et frôle le guide touristique. Dans la soirée thématique d'Arte intitulée "On se la coule douce", un documentaire germano-brésilien de S. Köster (1997) est traité en journal intime et en mode énonciatif direct. Avec son amie Jamili, danseuse de samba qu'il a rencontrée lors d'une traversée du Brésil, le narrateur allemand qui commente tente d'accomplir son rêve: changer de vie, plaquer l'Allemagne, s'installer au Brésil en bord de mer, sous le soleil et les palmiers, ouvrir un bar et le tenir avec sa compagne. Alors qu'il pense accomplir son rêve, il brise peu à peu celui de sa compagne, qui elle désire retourner en Allemagne, et donc quitter le Brésil où elle reste prisonnière des ses proches, de ses racines, de sa vie passée misérable, d'une mère qui l'a jadis abandonnée. On croit assister en direct à l'aventure, dans le cadre d'un journal au jour le jour, filmé sur un effet de mode énonciatif direct.

Lorsque la représentation télévisuelle s'applique à des objets dont la transformation est réglée par des programmes d'actions, des

1 Télérama, $\mathrm{n}^{\circ} 2278,8$ septembre 1993, p. 70.

2 Il faudrait examiner dans la même perspective les autres œuvres de Karlin et Lainé comme L'Amour en France (1990) ou Justice en France. 
algorithmes qui permettent de passer d'un état initial à un état final, on se trouve en face de la relation. Celle-ci se distingue de la chronique en ce qu'elle propose une unité logico-causale. Mais elle se distingue aussi de la séquence narrative du récit : elle se veut relation fidèle d'une suite d'actions quand le récit tend à faire passer un sens, poussé qu'il est par la visée de complétude. Les émissions télévisées de cuisine, la relation historique, les jeux télévisés, les séquences ludiques des bêtisiers, sketches comiques, Animo-gag ou Vidéo-gag, en offrent de claires illustrations.

Reste à examiner, au registre des séquences actionnelles les plus structurées, la séquence narrative, en justifiant d'abord les raisons qui poussent à une théorie restreinte de la narrativité. Il peut sembler, a-ton dit, plus judicieux de passer de la distinction descriptif / narratif à une échelle comprenant décrire, dépeindre, rapporter, relater, raconter. Or, la tendance à assimiler toute représentation d'actions à un récit a marqué longtemps l'ensemble de la sémio-narratologie (Todorov, Bremond, Genette, Ricœur, Greimas, Gaudreault, Metz). Ainsi, dans sa théorie générale de la signification, la sémiotique greimassienne (l'École de Paris) conjoint théorie sémique et théorie narrative. Les structures narratives sont alors placées au centre de la production de sens. On prolonge ainsi Levi-Strauss et Barthes. On place la narrativité au niveau de surface comme au niveau profond, on la libère des formes figuratives, on en fait le principe organisateur de tout discours et on homogénéise tout. Tout texte -politique, scientifique, juridique- devient narratif et tout niveau -énoncé, énonciationaussi. On narrativise la recette de cuisine, les passions, les questions conversationnelles et pragmatiques ${ }^{1}$. $\mathrm{Si}$, dans sa sémantique de l'action, P. Ricceur corrige sur plus d'un point la conception greimassienne d'une narrativité intrinsèque, s'il ajoute une dimension praxique et une relation entre ouvre et contexte, il maintient cependant le récit au rang de méta-genre et l'activité configurante "au rang d'intelligence narrative" , et il définit la mise en intrigue de manière très large. On trouve dans la sémio-narratologie immanentiste du cinéma des extensions comparables qui font de tout plan d'image

1 Un bilan critique de la sémiotique greimassienne et des propositions de Ricœur est apporté par J. Brès, La narrativité, Louvain-la-Neuve, Duculot, 1994.

2 P. Ricceur, "Une reprise de La Poétique d'Aristote", Lectures 2, Paris, Éd. du Seuil, 1992, p. 464-478. 
animée un récit et de la narrativité une propriété intrinsèque au plan' ${ }^{1}$. F. Jost en a proposé une critique judicieuse ${ }^{2}$. Il faut le suivre lorsqu'il invite à reformuler la problématique de la narrativité en dépassant le niveau de l'immanence pour intégrer les dimensions énonciatives et pragmatiques :

les transformations et les successions, la présence d'événements ne suffisent pas à la définir (la narrativité); elle ne commence que dans les documents où s'ajoute à ces observations la conviction que le temps n'est pas subi, mais voulu, intentionnel, en un mot humain : c'est en gros la frontière que marque le direct préparé, avec ses alternances de série construisant une simultanéité et ses mini-analepses que sont les replays ${ }^{3}$.

Il y a alors pour lui un en deçà de la narrativité (direct préparé et documentaire), une plénitude narrative (fiction) et un au-delà (film comme œuvre). Il faut encore suivre F. Jost lorsqu'il invite à fracturer le concept unique de narration (L'œil-caméra). Cependant, la prudence commande de mieux définir la description audiovisuelle et de reprendre avec précaution le projet metzien de "grande forme anthropologique" en ce qui concerne le récit. La télévision invite précisément à cela. Il est exact, comme le dit encore Jost, que "la narrativité ne s'exprime qu'à partir du moment où l'on peut déceler une intentionnalité non seulement discursive, mais narrative"4. Ajoutons qu'il faut encore que l'intentionnalité narrative déclarée se trouve effectivement concrétisée. Prenons l'exemple de la collection de Michel Serres et Robert Pansard-Besson La légende des sciences ${ }^{5}$. Dans ce cas, l'intention narrative est affichée : "si je parle de la science à des non spécialistes, je vais utiliser le récit" ou encore "chacun de ces films est l'occasion de raconter que l'explication intelligente du monde date de très longtemps, que c'est une longue

\footnotetext{
1 A. Gaudreault, Système du récit filmique, Paris, Méridiens-Klincksieck, 1988, p. 39. C. METZ, Essais sur la signification au cinéma, Paris, Klincksieck, 1968. Voir "Remarques sur une phénoménologie du narratif". C. METZ, Langage et cinéma, 1971.

2 F. Jost, "Propositions pour une typologie des documents audiovisuels", Sémiotica, $n^{\circ} 112,1 / 2,1996$, p. 123-140. Voir aussi "L'image fixe dans l'image animée", Littérature $80, \mathrm{n}^{\circ}$ 106, juin 1997, p. 80-88.

3 F. JosT, "Propositions...", op. cit., p. 136-137.

4 Ibid., p. 131.

5 Collection de douze émissions thématiques diffusées sur Arte (1997) et que Michel Serres présente comme son testament (Télérama, $\mathrm{n}^{\circ} 2474$, p. 114).
} 
coulée historique qui unit les peuples"'. En mobilisant la mythologie et les légendes, en visant le récit "étiologique" et exemplaire, il s'agit de tenter de raconter l'épopée de la science. Mais, si l'on regarde de près les films - par exemple le douzième consacré à l'astronomie- on ne retrouve pas à l'évidence les ambitions dites. Le lacis audiovisuel propose bien deux séquences narratives, fictionnelles et encadrantes, mais surtout plusieurs séquences essentiellement descriptives (observatoires du Monde, figurations dans le désert, voûte céleste...) et quelques séquences conversationnelles sur un plateau abstrait. Le commentaire des deux orateurs mêle les périodes descriptives, explicatives, argumentatives et poétiques. Qu'est devenue alors, dans cet ensemble résolument hétérogène, l'intention narrative ?

Il convient donc de reformuler plus strictement le narratif et la séquence narrative. Idéalement, aux trois niveaux hiérarchiques de l'acte de discours, de la séquence, de l'agir global, il est intentionnalité narrative qui déploie une mise en intrigue comportant une tension, sur l'axe nœud / dénouement. Le critère de la seule mise en intrigue ne suffit pas. Certes, il comporte l'opération synthétique de configuration et d'homogénéisation. Mais, au plan de la structuration du procès transformationnel, il faut encore installer le jeu du couple nœud / dénouement. En somme, à la notion strictement compositionnelle de mise en intrigue, il convient d'ajouter la notion sémantique de mise sous tension ${ }^{2}$, qui empêche d'en rester à la simple composition linéaire. On précisera encore deux points. La phase de l'action peut être remplacée par des données psychologiques rattachables aux modalités. D'autre part, qu'elle soit factuelle ou fictionnelle, la séquence narrative peut reproduire des scripts, ces cadres sociaux et cognitifs stéréotypés dont le statut est quelque peu problématique ${ }^{3}$, alors même que leur présence peut être effective. Au total, il paraît plus fructueux d'écarter les positions maximalistes du "tout est récit" ainsi que les affirmations qui en relèvent : tout texte d'action est récit; toute succession, juxtaposition, connexion est récit ; tout advenir est récit ; tout est surdéterminé en permanence par un

1 lbid.

2 Sur les bases évoquées, F. Revaz reformule le modèle quinaire du récit retenu par J.-M. Adam (après d'autres), en glissant aux deux bornes de la transformation (séquences 2 à 4), le nœud (avant la séq. 2) et le dénouement (après la séq. 4).

3 Pour Barthes, le script est récit; voir "Introduction à l'analyse structurale des récits", Communications, n 8 , 1966, p. 19-20. Pour U. Eco, il ne l'est pas : Lector in fabula, 1985, p. 140. 
grand récit, etc. Au niveau intermédiaire de la séquence, il est donc préférable de s'en tenir à une structuration de la mise en intrigue exigeant la mise en tension.

\section{La séquence explicative}

Considérons la collection documentaire de J.-L. Portron Paysages $^{1}$, plus particulièrement le numéro consacré à AuxeyDuresses (Côte d'Or). Le propos consiste à nous procurer un atlas des paysages par un ensemble de "lectures" qui saisiront dans l'apparence immédiate les indices de spécificité à décrypter. Le plus souvent, le numéro est découpé en strates séparées par un fondu au noir, et relancées par la répétition incessante d'un plan de référence du paysage examiné, le plan qui offre le meilleur point de vue. Chaque étape ou strate apporte la réponse à une question posée explicitement ou non. Ainsi : pourquoi cette concentration du vignoble en Côte d'Or, le long d'une étroite bande de $50 \mathrm{~km}$ de long ? Pourquoi ce morcellement des parcelles à Auxey-Duresses? Pourquoi cet ordre dans la culture de la vigne ? Pourquoi cette hiérarchie dans la valeur des parcelles? Quelles raisons font un grand vin? Les réponses à chaque question passent par le recours aux témoignages, cartes, plans, vues satellite, vues aériennes, croquis, photos, analyses à la palette graphique. Il s'agit donc par tous les moyens, y compris les extraits d'actualités d'époque, de traquer le paysage et le passé pour faire remonter à la surface l'invisible. La continuité audiovisuelle multiplie les plans descriptifs, s'autorise quelques séquences actionnelles discrètes (un train passe, un ouvrier brûle des ceps, une caméra subjective simule une balade en montagne) et quelques interventions monologales enchâssées. Dans son rapport au commentaire, l'asynchronie est dominante. Bref, le paysage se trouve expliqué jusqu'au secret qu'il recèle. Ainsi, Etretat finit par livrer sa guerre secrète entre les autochtones (maisonnettes à l'abri du vent), la jet-set parisienne (manoirs avec vue sur la mer), les deux ligués contre l'envahisseur (le touriste sur la falaise). Une leçon de géographie humaine nous est donnée, moyennant les conseils de sociologues, ethnologues, agronomes. Elle avoue une attention fervente au réel et

1 Il s'agit d'une collection de documentaires diffusés d'abord en 1993 (Fos sur Mer, Bierre lès Semur, Treis Garden, La Porte de Bagnolet, L'Ombrie : Campello Alto), puis en 1997 (Auxey-Duresses, Lorient, Etretat). 
aux gens qui témoignent, sans jamais basculer dans la démonstration lourde, même si elle n'exclut pas au passage le plaidoyer militant (la défense de Lorient). On se prend à penser que le paysage est traité comme un "lieu de mémoire", une "unité significative, d'ordre matériel ou idéel, dont la volonté des hommes ou le travail du temps a fait un élément symbolique d'une quelconque communauté" (Grand Robert). Un travail d'égale qualité est fait par A. Jaubert dans Palettes, émission consacrée à la "lecture de toiles". Avec un dispositif différent fondé sur les archives d'époque et l'analyse conversationnelle dialogale, Marc Ferro échafaude L'Histoire parallèle de la seconde guerre mondiale, en s'engageant de plus dans des comparaisons avec le présent.

Dans ces quelques exemples, on repère aisément la structuration de la séquence explicative : sur une schématisation initiale, un questionnement survient, il est résolu par une explication-réponse et clos par une conclusion-évaluation. Distincte du simple discours informatif et expositif, la séquence explicative est portée par la volonté de faire comprendre un problème, implicite ou explicite. En ce sens, tout paysage sélectionné est un ensemble problématique à élucider, qui en constitue le contrat implicite. En somme, la séquence explicative dynamise la séquence descriptive et la restructure autour de l'enchaînement question / réponse . Bien sûr, ladite séquence peut se faire elliptique, s'insérer dans la séquence descriptive ou narrative, comme elle peut se développer en séquence narrative. On peut parfois douter de la capacité de la télévision, au niveau de l'agir téléologique, à expliquer le monde, mais on ne peut douter de l'existence de séquences explicatives.

\section{Les séquences performatives}

Cette dernière catégorie de séquences élémentaires peut se subdiviser en séquences implicatives et séquences argumentatives. Au titre des premières, on citera les émissions du téléachat comme Téléshopping (TF1) et M6 Boutique, ainsi que les émissions de mobilisation des spectateurs autour d'une grande cause-Téléthon, Sidaction-, partout où agir axiologique, affectuel et spectaculaire interviennent. Au chapitre des secondes, on rangera maints documentaires et magazines qui s'organisent en démonstrations : tel épisode des Mercredis de l'Histoire (A. Adler), par exemple "Hitler : un 
inventaire" (1995) où, au fil de six numéros, on démontre comment Hitler est arrivé au pouvoir et a décervelé la société allemande; ou tel épisode des Brûlures de l'Histoire (P. Rotman, F3), par exemple "La nuit des longs couteaux", où il s'agit de démontrer comment Hitler a renforcé son pouvoir personnel. Actualités d'époque, films de propagande et commentaires d'historiens servent alors à apporter une leçon d'histoire.

Le schéma prototypique de l'édifice argumentatif est fondé sur la mise en relation de données avec une conclusion. Le segment étayant des données qui peuvent être fondées explicitement ou implicitement, contrariées ou confortées, sert ainsi d'argument(s) ou de raison(s) au segment étayé de la conclusion. Si on veut compliquer la démarche évoquée, on dira qu'à partir d'une éventuelle thèse antérieure, il s'agit d'avancer trois macro-propositions : des données servant de prémisses (prop. 1) ; des garants qui vont étayer ou seront nuancés selon des exceptions, restrictions, réfutations (prop. 2); une conclusion (prop. 3). Évidemment, on peut opter pour un ordre progressif (des données vers la conclusion) ou un ordre régressif (de la conclusion vers les données). Enfin, la séquence argumentative peut être narrativisée, comporter des moments descriptifs et explicatifs, ou relier argumentativement deux séquences descriptives.

Soit l'émission de la Télévision suisse romande Complètement télé (20-07-97, La Cinq). Le propos est de faire réfléchir au traitement télévisuel du fait divers "périlleux atterrissage à Genève d'un avion victime d'un accident de bord". Le dispositif est celui du Téléjournal, avec un présentateur introduisant les trois étapes de la démonstration et livrant la conclusion. L'ordre est progressif. Les données servant de prémisses constituent le reportage de départ, traité selon les conventions actuelles: images d'actions de sauvetage, témoignage d'un membre de l'aéroport de Genève, bilan provisoire et prudence du commentaire qui s'achève sur la question : attentat ou dysfonctionnement? Dans une deuxième phase introduite par le présentateur qui avertit que l'on va revoir la même séquence traitée de "manière plus spectaculaire", un certain nombre de garants vont être apportés dans le sens de la dramatisation : multiplication de caméras subjectives et de témoignages de passagers sous le choc, infléchissement du commentaire vers le mode subjectif fort, sélection d'un témoignage clé qui induit clairement l'hypothèse du terrorisme. Dans la dernière phase, précédée par le discours engagé du présentateur fier de montrer en exclusivité des images de caméra amateur qui font scoop, on voit 
ce qui se passe à l'intérieur de l'appareil. Mais, une réfutation soudaine surgit: "En réalité, cet incident ne s'est jamais produit" ! On nous explique que l'ensemble a été simulé et joué avec la complicité des autorités concernées. On nous laisse alors trouver la conclusion qui s'impose sur l'information-spectacle.

\section{Le réglage de l’hétérogénéité télévisuelle}

L'analyse qui vient d'être conduite a cherché à montrer que toute émission de télévision est faite de l'utilisation d'un nombre finalement assez restreint de séquences élémentaires inscrites dans des dispositifs à visées stratégiques. On pourrait en inférer un peu vite qu'il se passe pour ce média ce qui existe dans tout autre texte, littéraire par exemple. Or, cela n'est pas et il faut à présent déboucher sur les questions, et du fonctionnement spécifiquement télévisuel de la combinatoire séquentielle, et sur les particularités de ses modes de réglage.

\section{Le réglage de l'hétérogénéité programmatique}

Conformément au paradoxe déjà relevé par $\mathrm{E}$. Veron ${ }^{1}$, les médias sont habités par la dialectique de l'uniformisation / diversification. La concurrence économique pousse à la première, la quête d'identité porte à la seconde. Sévit alors une double contrainte : "homogénéisation des produits tout en ayant un besoin absolu de mise en valeur des différences". Si l'on entend rester dans la perspective de complexité retenue, il convient alors de ne pas réduire cette double contrainte à un seul des deux termes, le plus souvent l'homogénéisation par défaut. Mieux vaut parler de réglage de l'hétérogénéité.

Historiquement, et pour la télévision française, ce réglage a commencé à s'instituer en 1981, avec l'émergence du concept de marketing et la création du premier titre de directeur de la programmation (confié à P. Wiehn). Comme le dit M. Dagnaud, "le début des années quatre-vingt marque nettement un tournant dans la façon de

1 E. Veron, "Les médias en réception: les enjeux de la complexité", Médiaspouvoirs, $\mathrm{n}^{\circ} 21$, janvier-mars 1991. 
penser l'offre d'images"', et après H. Bourges et P. Josèphe, on peut à présent parler de "métabolisme cathodique". Si bien que le rôle actuel de la programmation dans le réglage de l'hétérogénéité des programmes peut aujourd'hui se décrire comme allongement des écrans, organisation des grilles en agendas de rendez-vous, adaptation des formats et des genres aux cases horaires en fonction d'études de publics, harmonisation générale, engagement dans la starisation et la spectacularisation ${ }^{3}$.

Les séquences interstitielles des interprogrammes sont alors à considérer dans ce cadre. Il s'agit en gros de séquences promissives (bandes-annonces et semainiers) et de séquences performatives, à la fois implicatives (publicité institutionnelle) et esthétiques (publicité commerciale). Elles sont autant de systèmes semi-ouverts qui fonctionnent, soit en germes, cellules ou matrices des programmes à venir, soit en espaces lucratifs à enjeu économique. S'il est vrai que le flux où ces séquences surgissent est marqué par la tension, il serait excessif d'aboutir à l'idée d'une uniformisation générale des interstices et, au-delà, de l'ensemble des programmes.

\section{Le réglage de l'hétérogénéité textuelle}

Comment définir alors, dans la perspective compositionnelle retenue, le texte souvent complexe de l'émission de télévision ? Il est fréquemment introduit ou annoncé par une séquence interstitielle promissive (au moins). Il est présenté par des séquences phatiques d'ouverture et de clôture, séquences conversationnelles monologales. Certains textes télévisuels sont unitypes et homogènes, formés d'une seule séquence qui, déclinée d'émission en émission, ne repose pas toujours sur la même visée pragmatique. La plupart sont donc pluritypes et hétérogènes, si bien que l' émission courante combine les séquences prototypiques élémentaires qui ont été décrites cidessus. Les critères de passage d'une séquence à l'autre reposent sur la disjonction thématique (changement de section et de rubrique), actorielle (changement d'invité ou d'actant), sémantique (changement de monde), générique (changement de format). Les trois grands

1 M. DaGnaud, "Profession programmateur", Médiaspouvoirs, $\mathrm{n}^{\circ} 20$, octobre-déc. 1990.

2 Y. Bourges et P. JosÉPHe, Un amour de TV, Paris, Plon, 1989.

3 M. DaGnaud, "L'âme des chaînes", Paris, CSA, 1991. 
modes de combinaisons sont la reprise-reformulation, la succession par juxtaposition ou coordination, l'intégration-enchâssement. Un texte télévisuel peut alors comporter une séquence, $\mathrm{n}$ séquences de même type ou de types différents parmi lesquelles s'installe un principe de dominance.

Dès qu'on parle de réglage de l'hétérogénéité, on postule qu'interviennent des principes qui paraissent devoir être rangés, moins dans le cadre rigide de conventions ou lois que dans le cadre relativement unifiant du dispositif stratégique ${ }^{1}$. Dans les discours stéréotypés qui circulent, le dispositif est souvent réduit à la dimension matérielle, technique (le plus souvent) et économique (parfois). Il faut bien comprendre qu'avec la télévision, nous sommes contraints d'envisager aussi le niveau symbolique d'une part, le niveau transtextuel d'autre part. Car la production télévisuelle comporte des émissions qui, vingt ans durant, peuvent se répéter (mise en série) tout en infléchissant leur agir dominant et donc leurs structurations. Et ce niveau à la fois paradigmatique et inter ou transtextuel nous oblige, de plus, à considérer l'existence de séquences réflexives dans ces émissions où la télévision se met au miroir, se regarde avec sérieux ou avec humour, renouvelle ses textes en méta-communiquant.

Par ailleurs, la notion de genre risque d'être un paravent commode, mais qui induit en erreur en poussant la réflexion presque automatiquement vers l'homogénéité. Or, prenons un exemple parmi d'autres, celui des magazines scientifiques, adulés dans les années 60 , abandonnés dans les années 70 , à nouveau courtisés dans les années 90. Un même genre, mais des dispositifs très variés, et une prédilection pour certaines séquences qui répondent à la visée stratégique du dispositif : les unes privilégient les exploits scientifiques spectaculaires quand les autres éclairent avec sérieux les phénomènes et les découvertes.

Enfin, quand l'on change de régime textuel, on change aussi de planification pour superposer aux séquences élémentaires un régime esthétique (J.-C. Averty, les expériences de l'INA dans les années 1975). Et cette esthétique télévisuelle mérite à elle seule un examen attentif.

1 Je renvoie ici à mon intervention "Les dispositifs télévisuels", colloque de Cerisyla-Salle "Penser la télévision", juin 1997, à paraître. 
Dans la perspective sémio-pragmatique qui a été adoptée, l'émission télévisuelle apparaît comme un ensemble textuel structuré en différents plans d'organisation, dont celui de l'organisation séquentielle. L'émission y est considérée comme une configuration hiérarchisée et complexe. La séquence, unité élémentaire, y est toujours prédication qui a une configuration pragmatique, à la fois sémantique, énonciative et illocutoire. Elle est structure ou réseau relationnel hiérarchique, relativement autonome et pourtant en relation avec l'ensemble plus vaste qui la contient. En ce sens, elle est constituée et constituante. La combinaison séquentielle apparaît réglée à différents niveaux : interne (liage), externe pragmatique (l'agir), externe programmatique (ligne éditoriale), externe générique (contraintes culturelles). En ce sens, l'hétérogénéité fondamentale de toute émission est relativisable.

Comme il a été affirmé, la séquence narrative n'est, à la télévision, qu'une séquence parmi d'autres. Bien sûr, la narrativité peut s'appréhender aux trois niveaux de surface du micro-acte, de la séquence, du texte tout entier, et même au niveau profond, plus idéologique et inconscient, du récit exemplaire. Mais il en va de même pour les autres actes illocutoires identifiés. Et si parfois, dans certains cas exceptionnels, la séquence peut être confondue avec le dispositif ou le genre, le plus souvent, la télévision a la capacité de pouvoir incruster en permanence les séquences, les dispositifs et les genres les uns dans les autres. Ce laboratoire de la séquentialité débouche donc sur la richesse des combinatoires hypertextuelles. 